\title{
La televisión como espejo de la realidad del «bullying». Percepciones de los adolescentes sobre el programa «Proyecto Bullying»1
}

\author{
Patricia de Casas-Moreno( $\left.{ }^{*}\right)$, Mari Carmen Caldeiro Pedreira( $\left.{ }^{* *}\right)$, Luis M. Romero-Rodríguez $\left(^{* *}\right)$ \\ ${ }^{*}$ )Universidad Nebrija, $\left.{ }^{* *}\right)$ Universidad Santiago de Compostela, $\left({ }^{* *}\right.$ ESAI Business School, Universidad Espíritu Santo (Ecuador)
}

\begin{abstract}
RESUMEN
Si bien el acoso escolar (bullying) es un fenómeno de vieja data, no es menos cierto que en la actual sociedad de la información existen claros indicadores que ha sido sometido a mayor tratamiento comunicacional, ergo mayor atención social y científica. A su vez, el desarrollo de las nuevas tecnologías ha provocado que el acoso escolar aumente en las aulas con el uso de los medios digitales como redes sociales o las cuartas pantallas (smartphones y tablets). La presente investigación tiene como objetivo analizar comparativamente las percepciones de 40 adolescentes de entre 12 y 16 años de cuatro provincias españolas (Lugo, Pamplona, Huelva y Badajoz) sobre el programa «Proyecto Bullying» de la cadena «Cuatro» a través de entrevistas en profundidad. Los resultados demuestran que los adolescentes están concienciados con el problema del acoso escolar. Sin embargo, son muchos aun los que muestran atenuación y vulnerabilidad frente al problema, rehuyendo de la búsqueda de soluciones y manteniéndose al margen.
\end{abstract}

Palabras Clave: bullying, televisión, adolescentes, entrevistas en profundidad, alfabetización mediática.

\section{Television as a mirror of reality about bullying. Perceptions of adolescents about the «Bullying Project»}

\section{ABSTRACT}

Although bullying is a longstanding phenomenon, it is no less true that in the current information society there are clear indicators that have been subjected to greater communication treatment, ergo greater social and scientific attention. Likewise, the development of new technologies has caused school bullying to increase in the classroom with the use of digital media such as social networks or the fourth screen (smartphones and tablets). The present research aims to comparatively analyze the perceptions of 40 adolescents aged between 12 and 16 from four Spanish provinces (Lugo, Pamplona, Huelva and Badajoz) on the «Bullying Project» program of the «Cuatro» TV station through depth interviews. The results show that adolescents are aware of the problem of bullying. However, many are still those that show attenuation and vulnerability to the problem, avoiding the search for solutions and staying on the sidelines.

Keywords: bullying, television, adolescents, in-depth interviews, media literacy.

\section{Introducción}

Los medios de comunicación se han afianzado paulatinamente en un modelo emergente de educación en las familias y en las escuelas. La falta de una correcta actitud crítica sobre los efectos que se pueden desarrollar a través del uso inadecuado de las Tecnologías de la Información y la Comunicación (TIC) se ha convertido en un tema de gran interés para la comunidad científica. En este sentido, el presente estudio está basado en el análisis de los términos «bullying» $\mathrm{y}$ «ciberbullying», así como la progresiva concienciación y relevancia que mantienen las agresiones en los contextos escolares y las posibles intervenciones como métodos de prevención del acoso escolar (Garaigordobil y Oñederra, 2010). Debido al aumento de este fenómeno en los centros educativos, son varios los autores que han investigado sobre la violencia entre iguales, elaborando instrumentos de evaluación y propuestas de intervención y prevención adaptadas a las diferentes edades en las que se produce (Cerezo, Calvo, y Sánchez, 2011; Gámez-Guadix, Villa- George, y Calvete, 2014; Álvarez-García, Dobarro, y Núñez, 2015; Schultze-Krumbholz, y Scheithauer 2016).

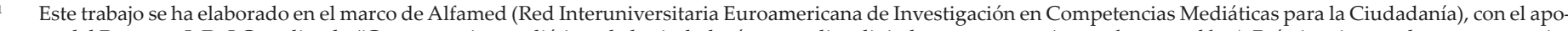

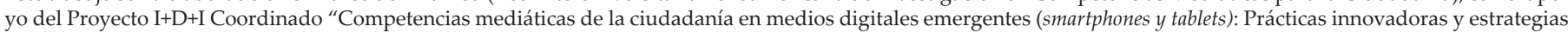

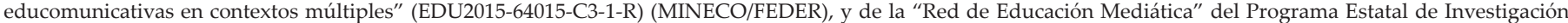

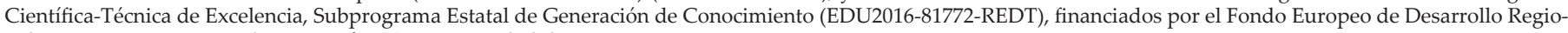
nal (FEDER) y Ministerio de Economía y Competitividad de España.
} 
Etimológicamente «bullying» es un término anglosajón que significa intimidación, maltrato y/o acoso, haciendo referencia a la existencia de una víctima indefensa, hostigada por un agresor cuyo objetivo es hacer daño de forma consciente. Según Lumsdem (2002) el «bullying» responde a una circunstancia en la que una persona se encuentra sometida por otra a través de la intimidación. Son muchos los autores que se han aventurado a arrojar una definición sobre este concepto pero sin duda, de una forma $\mathrm{u}$ otra, el término mantiene una carga negativa, independientemente del contexto socio-cultural donde se analice (Hernández-Granda, 2001; Nansel, Overpeck, Haynie, Ruan y Scheidt, 2003). Por su parte, el «ciberbullying», más arraigado en el ecosistema digital, consiste en realizar el mismo acto de acoso pero a través de las TIC como pueden ser las redes sociales o los teléfonos móviles (Garaigordobil, 2011; Olweus, 2013). Según Smith et al., (2008), el término de «ciberbullying» define a aquella conducta agresiva e intencional, repetida de forma constante por un individuo a través del uso de dispositivos tecnológicos en contra de una víctima sin medios para defenderse. Con el desarrollo de las TIC lo que conocíamos como acoso escolar o «bullying» ha pasado a llamarse «ciberbullying», ocupando las tecnologías un papel intermediario importante en la relación victimario-víctima. Frente a esta nueva tesitura, los investigadores demuestran la prevalencia desde sus inicios en 2002 hasta la actualidad, pasando de un $6,5 \%$ al $24,6 \%$ de incidencias de ciberacosos en los adolescentes (Ortega-Barón, 2016).

Los estudios sobre el fenómeno del maltrato entre iguales surgen en Europa a finales de los años 70 (Perry, Kusel, y Perry, 1988; Pepler y Rubin, 1991). En el caso español, los trabajos pioneros son aquellos relacionados con García-Orza (1995). Sin embargo, cabe señalar que a raíz del debate social que se produce en el siglo XXI, los estudios relacionados con el acoso escolar se multiplican a nivel nacional_(Ramírez, 2006). En la misma línea y con el desarrollo y popularización de las TIC, el término de acoso escolar ha comenzado a denominarse «ciberbullying». A pesar de ser reciente y con corta longevidad en los estudios, son muchas las investigaciones que se han realizado a nivel europeo, como por ejemplo las pesquisas realizadas por Reino Unido y España (Hinduja y Patchin, 2007, Smith et al., 2008; Avilés, 2009).

En la misma línea de estudio, los datos arrojados por las diferentes tesis señalan que se desarrollan acciones de intimidación, afectando entre un $14 \%$ y $18 \%$, adquiriendo especial notoriedad el género de los adolescentes, subrayando que el modus operandi más frecuente es el uso de rumores y aislamiento de la víctima por parte del sexo femenino; mientras que por parte del masculino se tiende más a las agresiones físicas y amenazas. De esta manera, esta diferenciación identifica el acoso directo con ataques abiertos a la víctima y el acoso indirecto, a través del aislamiento social al individuo. Seguidamente, en relación a la edad, prevalecen las víctimas de los dos primeros cursos de secundaria (12-14 años), mientras que en los dos últimos cursos (14-16 años), las cifras disminuyen (Romera et al., 2016). Por su parte, en el caso español, los estudios revelan que mantiene una proporción más elevada que la media europea frente al acoso escolar (ÁlvarezGarcía, Barreiro-Collazo y Núñez, 2017).

Otro aspecto importante sobre este tipo de acoso escolar es que se da principalmente a través de la exclusión social, el uso de motes o a través de la discriminación por motivos raciales o religiosos. Sin embargo, los investigadores suelen coincidir en que existe una serie de factores que ayudan a desencadenar este tipo de situaciones, como son: i) las relaciones con el grupo de pares, donde la amistad o el rechazo cobran un papel notorio; ii) los factores contextuales como las percepciones de los alumnos o profesores o incluso un mal funcionamiento en el núcleo familiar y; iii) el género de las víctimas y agresores (Vivar, 2010). Sin embargo, la problemática real de los adolescentes está arraigada en la conducta reiterada del abuso del poder, identificándose tres roles (Loredo-Abdalá, Perea-Martínez y López-Navarrete, 2008):

1. El acosador: se identifica con aquella persona que ejerce la violencia sobre un igual. Según los estudios realizados existe entre un $7 \%$ y un $9 \%$ de adolescentes que llevan a cabo este papel en las escuelas.

2. La víctima: se identifica con aquel individuo que muestra inferioridad por la falta de adaptación al contexto social. Los estudios señalan que alrededor de un $9 \%$ pertenecen a este grupo.

3. Los víctimas-perpetradores / las víctimas propiciatorias: son aquellos que desde su rol de victima evolucionan hasta convertirse en agresores. Existe entre un 3\% y un $6 \%$ de la población adolescente con estas características.

A raíz de la importancia que ha cobrado este fenómeno, de forma especial con el uso de Internet y sus variopintas plataformas -redes sociales, grupos de chat, mensajería instantánea, entre otros-; los medios de comunicación, sobre todo la televisión, se han convertido en modelos de prevención de la violencia a través del desarrollo de programas relacionados con la protesta de casos de acoso escolar, centrando especialmente su atención en la comprensión de la naturaleza destructiva para ambos protagonistas de las situaciones de «bullying». En este sentido, el uso de los documentos audiovisuales provoca un mayor impacto emocional y estimula la empatía con los individuos victimizados, favoreciendo este tipo de contenidos a los esfuerzos realizados en la educación sobre todo en las escuelas, superando los conflictos de enseñar en contextos culturales heterogéneos (Díaz-Aguado y Falcón, 2006).

En esta línea, diversos estudios coinciden al afirmar que las situaciones de agresión-victimización se producen a edades tempranas (Cerezo, 2006; Lacasa y Ramírez, 2017). Sin duda, la mejor forma de combatir el «bullying» o «ciberbullying» es la cooperación de todas las partes implicadas como son los profesores, padres, estudiantes y personal no docente de la institución educativa. Garaigordobil, Martínez-Valderrey y Machimbarrena (2017) propone cuatro niveles de intervención: en primer lugar, el institucional, donde el centro educativo debe asegurar su implicación frente al problema; en segundo lugar, el familiar, siendo necesaria la participación activa de los padres; en tercer lugar, el grupal, implantando programas de intervención socio-emocional y anti-bullying en las aulas; y por último, el individual, estableciendo un rol comunicador con el agresor y la víctima con ayuda de apoyo terapéutico.

En suma, puede afirmarse que los medios de comunicación, pese a no concebirse inicialmente con un papel formativo propio, se han convertido de forma especial a lo largo de los últimos años, en agentes formativos y mediadores responsables de la situación social que transmiten y elementos clave en el proceso de desarrollo de la mirada crítica (Aguaded-Gómez, Caldeiro-Pedreira y Rodríguez-López, 2015) del «prosumer» (Sánchez-Carrero y Contreras-Pulido, 2012) que hoy en día desarrolla un papel fundamental en la sociedad del espectáculo donde debe consumir contenidos audiovisuales de forma participativa y responsable (Hernández-Serrano, Renés-Arellano, Graham y Grenwill, 2017). Por ende, se considera que la alfabetización, entendida como la herramienta fundamental para empoderar al sujeto, es un medio efectivo para favorecer el desarrollo de la conciencia responsable, crítica y participativa. Además, más allá del desarrollo de la competencia digital (Intef, 2017) el profesorado debería 
trabajar para incluir en su quehacer diario temáticas relevantes que suscitan polémica y controversia, cuestiones que al mismo tiempo inciden de forma grave en la convivencia e interrelación social y ciudadana.

\section{Materiales y método}

El presente estudio tiene como objetivo analizar el rol de los adolescentes frente al acoso escolar y su repercusión en la cognición social de los mismos. Asimismo, se examinará cualitativamente, con alcance exploratorio, el contenido audiovisual y la influencia de éste a la hora de afrontar un problema tan asentado en la actualidad como es el acoso escolar, el «bullying» $\mathrm{O}$ «cyberbullying». En este contexto, la alfabetización mediática es una asignatura clave para conocer de qué manera pueden influir los mensajes y el contenido de los programas televisivos en los espectadores, sobre todo, en el público más vulnerable.

«Proyecto Bullying» nace con la idea de demostrar a la sociedad casos de acoso escolar y concienciar sobre este problema tan grave producido en los adolescentes. Este formato, cuya producción ha sido verdaderamente corta -contando tan solo con cuatro emisiones (marzo 2017) - por denuncias y problemas con la fiscalía de menores, ha generado un fuerte debate por el impacto emocional de sus imágenes y las declaraciones de los protagonistas. De esta manera, partiendo del formato audiovisual como modelo de prevención frente al problema de acoso escolar, este estudio pretende vislumbrar la respuesta del público más expuesto frente a estas situaciones a través de este formato televisivo.

\section{Participantes}

El universo de estudio está formado por estudiantes de primero a cuarto curso de Educación Secundaria Obligatoria (E.S.O) de los municipios Huelva (Andalucía), Badajoz (Extremadura), Lugo (Galicia) y Pamplona (Navarra). La extracción de la muestra ha seguido un muestreo intencional no probabilístico polietápico estratificado por conglomerados, utilizando cada institución como unidad muestral. La selección de los municipios en estudio se justifica en orden de obtener un muestreo de carácter exploratorio que permita realizar una comparativa de los entrevistados de las zonas norte y sur de la península. La selección de los centros se hizo de forma aleatoria dentro de cada estrato. Fueron gestionados los permisos correspondientes con los centros y la autorización de los padres, representantes y/o tutores.

La muestra quedó comprendida por 40 participantes de un total de 47 entrevistas recogidas, lo que arroja un porcentaje de participación efectiva $\left(\mathrm{P}_{\mathrm{e}}\right)$ de $85,10 \%$, indicativa de una excelente tasa de respuesta por criterio de Baxter y Babbie (2004). El criterio de selección primaria de la muestra efectiva $\left(\mathrm{PM}_{\mathrm{e}}\right)$ fue ubicar participantes que hayan visualizado al menos un episodio del programa «Proyecto Bullying» emitido por la cadena de titularidad privada «Cuatro».

Del total de 40 entrevistados, 20 corresponden al sexo femenino y 20 al sexo masculino (tabla 1). La muestra ha sido seleccionada de forma intencional en cuanto al sexo, con el objetivo de establecer una comparativa objetiva entre los distintos municipios españoles analizados. Según se extrae de la muestra efectiva, en relación al sector femenino las edades con mayor número de participantes son las jóvenes de 13 y 15 años, mientras que en el sector masculino, se repiten los jóvenes de 13, 15 y 16 años.

Tabla 1. Estratificación de los sujetos muestrales por edad, sexo y comunidad autónoma.

\begin{tabular}{|c|c|c|c|c|c|c|c|c|c|c|c|c|c|c|c|c|c|c|}
\hline \multirow{2}{*}{$\begin{array}{l}\text { Comunidad } \\
\text { autónoma / } \\
\text { edad / sexo }\end{array}$} & \multicolumn{3}{|c|}{12} & \multicolumn{3}{|c|}{13} & \multicolumn{3}{|c|}{14} & \multicolumn{3}{|c|}{15} & \multicolumn{3}{|c|}{16} & \multicolumn{3}{|c|}{ Total } \\
\hline & $\mathrm{M}$ & $\mathrm{F}$ & $\Sigma$ & $\mathrm{M}$ & $\mathrm{F}$ & $\Sigma$ & $\mathrm{M}$ & $\mathrm{F}$ & $\Sigma$ & $\mathrm{M}$ & $\mathrm{F}$ & $\Sigma$ & M & $\mathrm{F}$ & $\Sigma$ & $\mathrm{M}$ & $\mathrm{F}$ & $\mathrm{T}$ \\
\hline Andalucía & 0 & 0 & 0 & 0 & 2 & 2 & 1 & 2 & 3 & 2 & 1 & 3 & 2 & 0 & 2 & 5 & 5 & 10 \\
\hline Extremadura & 0 & 2 & 2 & 4 & 2 & 6 & 1 & 0 & 1 & 0 & 1 & 1 & 0 & 0 & 0 & 5 & 5 & 10 \\
\hline Galicia & 2 & 0 & 2 & 0 & 1 & 1 & 1 & 1 & 2 & 1 & 2 & 3 & 1 & 1 & 2 & 5 & 5 & 10 \\
\hline Navarra & 0 & 0 & 0 & 1 & 1 & 2 & 0 & 0 & 0 & 2 & 2 & 4 & 2 & 2 & 4 & 5 & 5 & 10 \\
\hline Total & 2 & 2 & 4 & 5 & 6 & 11 & 3 & 3 & 6 & 5 & 6 & 11 & 5 & 3 & 8 & 20 & 20 & 40 \\
\hline
\end{tabular}

\section{Instrumento}

Se construyó un cuestionario ad hoc tomando en consideración los objetivos del presente estudio. El instrumento fue previamente validado por juicio de expertos, arrojándose un porcentaje de acuerdo $k=>0,88$ y un grado de consistencia interna de 0,735 . La herramienta se ha conformado a partir de dos bloques diferenciados. Por un lado, se realizan preguntas de carácter socio-demográfico, incluyendo los datos personales de los entrevistados como el sexo, la edad, la localidad y la provincia, con el objetivo de limitar y establecer las comparativas de estudio. Por otro lado, en el segundo bloque se ha tratado la entrevista compuesta por 21 ítems, presentando una serie de indicadores como la comprensión del término «bullying» y el uso de las nuevas tecnologías; el rol del menor frente al acoso escolar; y el conocimiento y visionado del programa seleccionado. Las preguntas son de modalidad abierta con la finalidad de obtener amplios resultados sobre el objeto de estudio pero sin llegar a la sobresaturación informativa.

\section{Procedimiento}

Para la recogida de datos primero se contó con todos los permisos necesarios y consentimiento informado tanto al alumnado, como al centro y a las familias, cumpliendo con la normativa legal vigente en materia de protección de menores y de datos. Las entrevistas fueron desarrolladas in situ en cada uno de los centros entre el 5 y el 23 de junio de 2017 por investigadores del proyecto I+D+I Coordinado «Competencias mediáticas de la ciudadanía en medios digitales emergentes (smartphones y tablets): Prácticas innovadoras y estrategias educomunicativas en contextos múltiples» (EDU2015-64015-C3-1-R) (MINECO/FEDER), y de la «Red de Educación Mediática» del Programa Estatal de Investigación Científica-Técnica de Excelencia, Subprograma Estatal de Generación de Conocimiento (EDU2016-81772-REDT). 


\section{Análisis de datos}

Las entrevistas fueron codificadas con la ayuda de una tabla de categorías y dimensiones emergentes, resultantes de la compilación en familias hermenéuticas de lexicones, señalando los tópicos más relevantes de la investigación. Este proyecto se encuentra enmarcado en la configuración teórica del análisis del discurso, haciendo uso de las unidades hermenéuticas más utilizadas en los mensajes positivos y negativos del contenido audiovisual mediante el software MAXQDA (v. 12.0). El análisis del discurso de las entrevistas de esta investigación se fundamentan en los tres principios básicos señalados por van Dijk (1983): funcionalidad, significación y direccionalidad de la meta. En este sentido, la funcionalidad se enfoca en las opciones léxicas del discurso (lexicones); la significación se refiere a la estructura y coherencia en las oraciones sobre una temática en general; y la direccionalidad de la meta consiste en los actos inspirados en el discurso. De igual manera, indica que los principios no son excluyentes, justificando unidades informativas que componen varios principios del análisis semántico del discurso (Torres-Toukoumidis, Romero-Rodríguez, De-Casas-Moreno y Aguaded, 2017).

Tras realizar la comparativa de los códigos emergentes analizados, se han podido contabilizar un total de 1.220 sistemas de códigos examinados en las entrevistas. De este modo, la exposición de los datos queda representada de la siguiente forma, así como el número de códigos por dimensión:

1. Implicación y ajuste social con la víctima y/o agresor.

a. Afrontar y superación de los hechos.

b. Manifestación de vulnerabilidad de los hechos.

2. Diversificación del rol agresor/victima.

a. Convivencia con el rol de víctima.

b. Polarización con el rol de agresor.

3. Abordaje de la problemática de estudio desde el punto de vista audiovisual.

a. Enfatización por la búsqueda de soluciones.

b. Atenuación frente al acoso escolar.

4. Atribuciones ideológicas del contexto.

a. Valores negativos del término «bullying».

b. Valores positivos del término «bullying».

\section{Resultados}

A la hora de establecer una diferenciación entre los códigos analizados, se ha hecho hincapié en una disimilitud entre los códigos positivos (861) frente a los códigos negativos (359) en el discurso de los adolescentes. En este sentido, para comprender los resultados de la investigación, conviene proceder a exponer los datos analizados a través de la separación y desglose de los códigos creados. De esta forma, adquiere un valor relevante el análisis de contenido realizado a través del software MAXQDA que ha permitido cuantificar y cualificar las unidades informativas recolectadas. Por lo tanto, el análisis llevado a cabo a raíz del desarrollo de las 4 familias de códigos se presenta de la siguiente forma según sexo, edad y procedencia del participante:

\section{Implicación y ajuste social con la víctima y/o agresor}

El discurso analizado muestra un objetivo común: analizar y conocer la influencia del programa seleccionado sobre el público más vulnerable de la sociedad, donde actualmente el problema del «bullying/ciberbullying» está más arraigado. De esta forma, en relación al primer código, se aprecia un alto grado de aceptación en la dimensión de «afrontar y superación de los hechos» con
135 reiteraciones, frente al descenso sobre la «manifestación de vulnerabilidad de los hechos» con 64 códigos emergentes. Además, para ser más precisos con los resultados hallados se puede establecer una disimilitud entre el sexo de los adolescentes, demostrando que el sexo femenino consigue alcanzar las máximas reiteraciones a la hora de cuestionarlos con los problemas de acoso escolar en el aula. En este sentido, el sector femenino presenta un total de 92 códigos frente a los 43 del sector masculino en el momento de afrontar los hechos, estableciéndose diferencias significativas en base al género de los entrevistados. Por su parte, en relación a la manifestación de vulnerabilidad se han encontrado 39 códigos del sexo femenino y 25 del sexo masculino.

Por otro lado, en relación a la edad, los niños/as de 16 años han acaparado el mayor valor numérico con 38 códigos en lo concerniente a la dimensión positiva de los hechos, mientras que el menor valor se encuentra en aquellos que tienen 12 años. En lo referente a la procedencia, los pacenses han conseguido un total de 48 reiteraciones frente a los onubenses y lucenses con 27 y 28 códigos respectivamente. Al respecto de la dimensión negativa y características vulnerables del sujeto, el valor más elevado se ubica en aquellos adolescentes de 12 y 13 años, y la localidad que ha obtenido la mejor puntuación ha sido Lugo con 19 códigos repetidos (ver Tabla 2 ).

Tabla 2. Código: Implicación y ajuste social con la víctima y/o agresor.

\begin{tabular}{|c|c|c|c|}
\hline & & $\begin{array}{c}\text { Afrontar } \\
\text { y superación } \\
\text { de los hechos } \\
(135)\end{array}$ & $\begin{array}{c}\text { Manifestación } \\
\text { de } \\
\text { vulnerabilidad } \\
\text { de los hechos } \\
(64)\end{array}$ \\
\hline \multirow[t]{2}{*}{ SEXO } & FEMENINO & 92 & 39 \\
\hline & MASCULINO & 43 & 25 \\
\hline \multirow[t]{5}{*}{ EDAD } & 12 AÑOS & 14 & 18 \\
\hline & 13 AÑOS & 26 & 15 \\
\hline & 14 AÑOS & 23 & 13 \\
\hline & 15 AÑOS & 34 & 11 \\
\hline & 16 AÑOS & 38 & 7 \\
\hline \multirow[t]{4}{*}{ PROCEDENCIA } & LUGO & 28 & 19 \\
\hline & PAMPLONA & 32 & 12 \\
\hline & HUELVA & 27 & 18 \\
\hline & BADAJOZ & 48 & 15 \\
\hline
\end{tabular}

En este sentido, la muestra seleccionada y entrevistada certifica una apuesta marcada por afrontar el acoso escolar en el aula y resalta la búsqueda de soluciones para radicar esta problemática. El visionado del programa por parte de la muestra, deja en evidencia que el «bullying» se ha convertido en un tema importante, que debe ser tratado y exterminado de la escuela. Los adolescentes demuestran con sus afirmaciones el interés por el programa audiovisual y por la superación de los hechos, incidiendo en la colaboración entre compañeros, padres y profesores. Asimismo, se puede destacar como a medida que la edad desciende el planteamiento y el trato sobre esta problemática es afrontado de forma responsable, demostrando conciencia, propuestas y soluciones (ver Tabla 3).

\section{Diversificación del rol agresor/víctima}

En relación con la segunda familia de códigos, las respuestas analizadas inciden en «convivir con la víctima» (199), sin embargo señalan y atacan al agresor en su discurso, consiguiendo que el código de «polarización con el rol del agresor» (107) no presente diferencias significativas en la reitera- 
Tabla 3. Análisis cualitativo: Implicación con los roles.

\begin{tabular}{|c|c|c|}
\hline & FEMENINO & MASCULINO \\
\hline \multirow[t]{3}{*}{$\begin{array}{c}\text { Afrontar y superación } \\
\text { de los hechos }\end{array}$} & $\begin{array}{c}\text { «Sí. Les permite expresar sus sentimientos y } \\
\text { ven que hay gente a la que le importa lo que } \\
\text { les está pasando» (M16-P) }\end{array}$ & $\begin{array}{c}\text { «El programa ayuda a que conozcamos los } \\
\text { casos y podamos comprender y ayudar a } \\
\text { personas que esté sufriendo acoso escolar» } \\
(\mathrm{H} 14-\mathrm{H})\end{array}$ \\
\hline & $\begin{array}{l}\text { «Sí, porque hace pensar a todo el público } \\
\text { que lo ve y esto ayuda a la hora de la verdad } \\
\text { a que no se queden con los brazos cruzados } \\
\text { (M15-B) }\end{array}$ & $\begin{array}{c}\text { «Parece un buen programa para ayudar } \\
\text { a comprender el problema del bullying» } \\
(\mathrm{H} 15-\mathrm{H})\end{array}$ \\
\hline & $\begin{array}{c}\text { «La impotencia y el sufrimiento de la persona } \\
\text { acosada y su familia» (M16-P) }\end{array}$ & $\begin{array}{c}\text { «Solo a veces porque se creen que son bromas } \\
\text { entre niños, otros profesores sí se lo toman } \\
\text { más enserio» }(\mathrm{H} 15-\mathrm{H})\end{array}$ \\
\hline $\begin{array}{l}\text { Manifestación } \\
\text { de vulnerabilidad } \\
\text { de los hechos }\end{array}$ & $\begin{array}{l}\text { «Bueno, conmigo antes se metían mucho } \\
\text { porque llevaba braques y me llamaban } \\
\text { dientes de hierro. Yo no les echaba mucha } \\
\text { cuenta pero había veces que me sentía muy } \\
\text { mal de las veces que lo repetían» (M14-H) }\end{array}$ & $\begin{array}{c}\text { «Son casos muy preocupantes ya que el } \\
\text { acoso aumenta y a veces no se sabe quién es } \\
\text { la persona que está detrás de los mensajes» } \\
(\text { H14-G) }\end{array}$ \\
\hline
\end{tabular}

ción del discurso. Asimismo, en el momento de evidenciar los datos según el sexo de la muestra se puede indicar que de forma reiterada, las respuestas del sexo femenino son más contundentes en sus respuestas. La víctima de los constantes acosos se convierte en el símbolo de empatía para el sector femenino con un total de 102 códigos frente a los 97 del sector masculino. A pesar de no ser muy significativas las diferencias, las jóvenes, apuestan por proteger y sentir aprensión por sus compañeros agredidos. Por su parte, en relación con la polarización con el agresor, los datos se invierten, convirtiendo al sector masculino, con 69 códigos, en el sector que más incrementa su conteo en las respuestas, mientras que el sector femenino asciende a 38 códigos.

En el tema relacionado con la edad, hay que señalar que los niños/as de 12 y 13 años presentan una empatía más próxima con la víctima, alcanzando 52 códigos. Además, Badajoz se convierte en la localidad con más reiteraciones positivas con un total de 55 frente a las 41 de Pamplona. Mientras tanto, a la hora de hablar sobre la polarización del agresor, los sujetos con más reiteraciones son los 15 años con 29 códigos y la localidad más significante es Huelva con 38 códigos frente a los 21 códigos de Pamplona (ver Tabla 4).

Tabla 4. Código: Diversificación del rol agresor/víctima.

\begin{tabular}{cccc}
\hline & & $\begin{array}{c}\text { Convivencia con el rol de } \\
\text { víctima (199) }\end{array}$ & $\begin{array}{c}\text { Polarización con el rol de } \\
\text { agresor (107) }\end{array}$ \\
\hline \multirow{2}{*}{ SEXO } & FEMENINO & 102 & 38 \\
& MASCULINO & 97 & 69 \\
& 12 AÑOS & 48 & 21 \\
& 13 AÑOS & 52 & 18 \\
& 14 AÑOS & 31 & 24 \\
PROCEDENCIA & 15 ANOS & 35 & 29 \\
& 16 AÑOS & 33 & 15 \\
& LUGO & 51 & 25 \\
& PAMPLONA & 41 & 21 \\
& HUELVA & 52 & 38 \\
& BADAJOZ & 55 & 23 \\
\hline
\end{tabular}

En este sentido, se apuesta por la empatía con la víctima y además, se hace hincapié en las actuaciones injustificadas de los agresores y sus motivos. Las diferencias significativas que se pueden observar en las edades de los entrevistados, demuestran que aquellos con edades entre medias, comparten sus pensamientos sobre el acoso, mientras que los más mayores se quedan en un segundo plano. Por su parte, en lo referido con la procedencia, el sur español se alza con la máxima puntuación. A continuación y para facilitar la comprensión de los resultados puede consultarse la Tabla 5, que contiene los datos relativos a la diversificación de roles: 
Tabla 5. Análisis cualitativo: Diversificación de roles.

\begin{tabular}{|c|c|c|}
\hline & FEMENINO & MASCULINO \\
\hline \multirow{2}{*}{$\begin{array}{c}\text { Convivencia con el rol de } \\
\text { víctima }\end{array}$} & $\begin{array}{l}\text { «Lo mal que se puede llegar a sentir una } \\
\text { persona porque un compañero le diga cosas } \\
\text { que no son verdad» (M14-B) }\end{array}$ & $\begin{array}{l}\text { «Entre compañeros de clase debemos } \\
\text { protegernos y respetarnos» }(\mathrm{H} 15-\mathrm{H})\end{array}$ \\
\hline & $\begin{array}{l}\text { «Me identifico un poco con las víctimas» } \\
(\text { (M14-H) }\end{array}$ & $\begin{array}{c}\text { «Hablar con ellos, apoyarlos consolarlos» } \\
(\text { H12-B) }\end{array}$ \\
\hline \multirow{2}{*}{$\begin{array}{l}\text { Polarización con el rol de } \\
\text { agresor }\end{array}$} & $\begin{array}{l}\text { «Que es mala persona y que no sabe el daño } \\
\text { que le hace a quien le acosa» (M13-G) }\end{array}$ & $\begin{array}{l}\text { «Que es una manera para que el acoso } \\
\text { escolar esté activo las } 24 \text { horas del día, ya que } \\
\text { pueden meterse con esa persona fuera del } \\
\text { colegio sin tener que verles la cara» }(\mathrm{H} 16-\mathrm{H})\end{array}$ \\
\hline & «Están enfermos» (M15-P) & $\begin{array}{l}\text { «Pues que es gente mala, que no tiene cosas } \\
\text { importantes que hacer, solo saben insultar a } \\
\text { las personas que les cae mal» (H14-B) }\end{array}$ \\
\hline
\end{tabular}

\section{Abordaje de la problemática de estudio desde el punto de vista audiovisual}

En cuanto a la tercera familia de códigos, destaca la «enfatización por la búsqueda de soluciones» que mantiene una posición notoria con 303 códigos, frente a los 185 códigos que presenta la «atenuación frente al acoso escolar». En relación con la diferencia según el sexo, las respuestas de las chicas alcanzan 165 códigos frente a los 138 códigos de los chicos. De esta manera, se evidencia que las jóvenes, se posicionan a favor de la víctima, ayudando y colaborando para erradicar esta situación frente a la disposición que mantienen los jóvenes. Por otro lado, en el momento de señalar la atenuación o mitigación de los entrevistados, se revela que el sector masculino con 105 códigos, es más favorable a expresar su temor por el acoso escolar frente a los 80 códigos del sector femenino, incidiendo en las pocas posibilidades de mejoras por miedo a las repercusiones directas sobre ellos.

Por otro lado, los adolescentes que con más frecuencia buscan soluciones ante el problema son aquellos con 15 años con un total de 73 códigos y la población con mayor puntuación es Badajoz con 92. Sin embargo, frente a la atenuación de los jóvenes, aquellos con 14 años son los que alcanzan los máximos valores con 50 códigos, mientras que los sujetos de 12 años obtienen el valor menos elevado con 30 códigos. En lo concerniente con la procedencia, Lugo obtiene un total de 57 reiteraciones, mientras que Pamplona alcanza tan solo 35 códigos (ver Tabla 6).

Tabla 6. Código: Abordaje de la problemática de estudio desde el punto de vista audiovisual.

\begin{tabular}{cccc}
\hline & & $\begin{array}{c}\text { Enfatización por la búsqueda } \\
\text { de soluciones (303) }\end{array}$ & $\begin{array}{c}\text { Atenuación frente al acoso } \\
\text { escolar (185) }\end{array}$ \\
\hline \multirow{2}{*}{ SEXO } & FEMENINO & 165 & 80 \\
EDAD & MASCULINO & 138 & 105 \\
& 12 AÑOS & 57 & 30 \\
& 13 AÑOS & 43 & 43 \\
PROCEDENCIA & 14 ANOS & 71 & 38 \\
& 15 AÑOS & 73 & 35 \\
& 16 AÑOS & 59 & 39 \\
& LAMGO & 77 & 57 \\
& PAUELONA & 53 & 35 \\
& BADAJOZ & 81 & 43 \\
\hline
\end{tabular}

En este caso debe subrayarse que a la hora de realizar el análisis de contenido se ha observado que los entrevistados apuestan por la búsqueda de soluciones y destacan por ayudar a las víctimas del acoso escolar. Sin embargo, hay que señalar que éstos presentan ideas pero a la hora de llevarlas a cabo son más reacios debido quizás, a posibles repercusiones en primera persona que puedan sufrir por parte del «agresor». Asimismo se demuestra que el miedo y los valores negativos aumentan debido al sentimiento de no querer ser agredidos o insultados. Los resultados demuestran que en relación a la edad, los mayores son más proclives a presentar y a aportar soluciones e intentar remediarlos por ellos mismos. Sin embargo, según el lugar de procedencia se puede señalar que el norte español presenta la característica y la falta de conocimientos sobre los términos de «bullying» y sus variantes, presentando la necesidad imperante de ofrecer cursos de formación sobre este gran problema de actualidad en las aulas. Esta hipótesis se refleja en las siguientes declaraciones (ver Tabla 7): 
Tabla 7. Análisis cualitativo: Abordaje de la problemática.

\begin{tabular}{|c|c|c|}
\hline & FEMENINO & MASCULINO \\
\hline \multirow[b]{2}{*}{$\begin{array}{l}\text { Enfatización por la búsqueda } \\
\text { de soluciones }\end{array}$} & $\begin{array}{l}\text { «Mejor hacerlo así. Es difícil para esas } \\
\text { personas explicar su problema abiertamente» } \\
(\mathrm{M} 15-\mathrm{H})\end{array}$ & $\begin{array}{c}\text { «Pienso que hay que evitar dar de lado a los } \\
\text { compañeros menos sociables y hacer piña } \\
\text { frente a los matones» (H14-H) }\end{array}$ \\
\hline & $\begin{array}{c}\text { «Defenderle y decirle solamente que no se } \\
\text { metieran con él o con ella» (M13-B) }\end{array}$ & $\begin{array}{l}\text { «Hay que ser respetuosos con nuestros } \\
\text { compañeros y ayudar a la gente que se } \\
\text { encuentre en problemas» (H13-B) }\end{array}$ \\
\hline \multirow[t]{2}{*}{$\begin{array}{l}\text { Atenuación frente al acoso } \\
\text { escolar }\end{array}$} & $\begin{array}{l}\text { «La frialdad y normalidad con que actúan los } \\
\text { acosadores y sin tener motivo, es una manía» } \\
(\text { M14-G) }\end{array}$ & $\begin{array}{c}\text { «La gente está muerta de miedo y no solo los } \\
\text { niños también los padres» (H15-P) }\end{array}$ \\
\hline & $\begin{array}{l}\text { «Pienso que creen que los niños exageramos» } \\
(\mathrm{M} 15-\mathrm{H})\end{array}$ & $\begin{array}{l}\text { «Intento defenderlo mientras que no me } \\
\text { perjudique a mi» }(\mathrm{H} 15-\mathrm{H})\end{array}$ \\
\hline
\end{tabular}

\section{Atribuciones ideológicas del contexto}

En relación a los valores positivos y negativos extraídos del análisis del discurso, se puede resaltar que los «valores negativos sobre el término bullying» presentan una reiteración más elevada con un total de 224 códigos, frente a la insignificante cantidad que reciben los «valores positivos del término bullying» con tan solo 3 códigos. Por su parte, en el momento de indicar las diferencias por sexo, se puede destacar que en lo concerniente a valores negativos, el sector femenino ocupa un alto grado con 120 reiteraciones frente a las 104 del sector masculino, mientras que en los valores positivos, solo se han descubierto un total de 3 códigos, pertenecientes al sexo masculino.

Los valores negativos analizados, diferenciados por edades, demuestran que los adolescentes de 15 años presentan el mayor número de repeticiones con un total de 68 , mientras que según la procedencia Huelva ocupa el lugar más elevado con la misma cantidad. Por su parte, los valores positivos hallados son casi inexistentes. Tan solo se han localizado 3 reiteraciones en sujetos de 15 años, perteneciendo 2 de éstos códigos a la localidad de Badajoz (ver Tabla 8).

Tabla 8. Código: Atribuciones ideológicas del contexto.

\begin{tabular}{cccc}
\hline & & $\begin{array}{c}\text { Valores negativos del término } \\
\text { «bullying» }(224)\end{array}$ & $\begin{array}{c}\text { Valores positivos del término } \\
\text { «bullying» (3) }\end{array}$ \\
\hline \multirow{2}{*}{ SEXO } & FEMENINO & 120 & 0 \\
& MASCULINO & 104 & 3 \\
& 12 AÑOS & 31 & 0 \\
& 13 AÑOS & 51 & 0 \\
PROCEDENCIA & 14 AÑOS & 32 & 3 \\
& 15 AÑOS & 68 & 0 \\
& 16 AÑOS & 42 & 0 \\
& LUGO & 53 & 0 \\
& PAMPLONA & 60 & 1 \\
& HUELVA & 68 & 2 \\
& BADAJOZ & 43 & 0 \\
\hline
\end{tabular}

De esta manera, se afronta la necesidad de reforzar en las aulas las pautas correctas para terminar con el acoso escolar y de qué manera, los más jóvenes pueden ayudar a los compañeros expuestos en este problema. Sin embargo, existe, aunque en un grado poco elevado, respuestas sesgadas que enfatizan al gran problema del acoso escolar, «bullying o ciberbullying», sobre todo, en el sector masculino, que se erigen como la muestra menos comprometida con la causa. A medida que se analizan las diferentes edades, se puede observar como los más mayores presentan un vocabulario y expresiones más relacionadas con sentimientos de odio y frustración con la causa (ver Tabla 9).

En relación con los diferentes códigos puede afirmarse que no existen diferencias notables entre la zona norte de España, representada por Pamplona y Galicia y la Sur, Huelva y Badajoz. En relación con la implicación y el ajuste social se aprecia que en la zona norte existe un número menor de entrevistados que afrontan y superan los hechos, un dato que coincide con la vulnerabilidad ante los mismos. En este último aspecto, en la zona sur en ambos casos se detecta un mayor número de respuesta lo cual confirma que el colectivo adolescente del sur de España posee un grado de implicación superior. Estas cifras coinciden con las referidas a la «Diversificación del rol agresor/ víctima».

Los códigos analizados referidos al abordaje de la problemática desde el punto de vista audiovisual coinciden con lo indicado hasta el momento. Sin embargo, en cuanto 
Tabla 9. Análisis cualitativo: Valores positivos y negativos.

\begin{tabular}{|c|c|c|}
\hline & FEMENINO & MASCULINO \\
\hline \multirow[t]{2}{*}{$\begin{array}{l}\text { Valores negativos del } \\
\text { término «bullying» }\end{array}$} & $\begin{array}{l}\text { se trata de hacer sentir mal a la gente, ofen- } \\
\text { diéndoles o haciéndoles sentir malas personas } \\
(\mathrm{M} 15-\mathrm{H})\end{array}$ & $\begin{array}{l}\text { El whatsapp, facebook o instragram se han } \\
\text { convertido en redes sociales para acosar e } \\
\text { intimidar. Muchos se hacen cuentas falsas } \\
\text { para meterse con la gente }(\mathrm{H} 14-\mathrm{H})\end{array}$ \\
\hline & $\begin{array}{l}\text { «Cualquier forma de maltrato psicológico, verbal } \\
\text { o físico producido entre escolares» } \\
\text { (M15-B) }\end{array}$ & $\begin{array}{l}\text { «Situación de maltrato o vejación a otra } \\
\text { persona» }(\mathrm{H} 14-\mathrm{G})\end{array}$ \\
\hline \multirow{2}{*}{$\begin{array}{l}\text { Valores positivos del } \\
\text { término «bullying» }\end{array}$} & ---- & $\begin{array}{l}\text { «No me ha impactado nada del programa ni } \\
\text { para bien, ni para mal» (H14-B) }\end{array}$ \\
\hline & ---- & $\begin{array}{l}\text { «No suelo ayudar a nadie que se encuentre } \\
\text { en problemas» }(\mathrm{H} 12-\mathrm{G})\end{array}$ \\
\hline
\end{tabular}

a las atribuciones ideológicas del contexto, se encuentran diferencias según referíamos a valores positivos o negativos del «bullying», a diferencia de los códigos anteriores, es mayor el número de entrevistados de la zona norte que hace referencia a los valores negativos del «bullying» y llama la atención que ningún adolescente haga referencia a valores positivos, por su parte, las respuestas de la zona sur indican que es menor el número de valores positivos que le atribuyen al «bullying» y en tres ocasiones hacen referencia a valores positivos del mismo. Siguiendo estos datos podemos señalar que los adolescentes de la zona norte consideran que el «bullying» constituye una lacra y no posee valor positivo alguno frente a los de la zona sur que, si bien señalan que es negativo, de forma minoritaria, le atribuyen algún valor positivo. Pese a todo es mayor el número de entrevistados de la zona sur que busca soluciones a tal problemática, lo cual confirma que se trata de adolescentes comprometidos que se solidarizan con las víctimas y apuestan por la superación de los hechos.

\section{Discusión y conclusiones}

Las TIC y los medios de comunicación se han erigido como los pilares básicos del entendimiento de los fenómenos sociales, no solo como elementos a través de los cuales se establecen relaciones interpersonales y herramientas comunicativas fundamentales, sino también como elementos clave en la formación del sujeto, especialmente los menores. Al mismo tiempo y en relación con los programas televisivos, es necesario subrayar la importancia de algunos como «Proyecto Bullying», espacios informativos que se convierten en demostraciones de la realidad. Más allá del formato o de la tipología de la programación en la investigación, se ha centrado la atención en los jóvenes de 13 a 16 años, chicos y chicas que día a día están en contacto con situaciones de acoso. Asimismo, las dimensiones de análisis, edad y procedencia de los adolescentes, se han convertido en una pieza clave para intentar demostrar las diferencias significativas frente al problema en el mapa español.

Este hecho, en la actualidad, se está convirtiendo en factor común y preocupa a los múltiples colectivos. En este sentido y debido al interés que despierta la terrible situación del acoso escolar, ya sea mediada por las tecnologías de la información o no, surge la inclinación por conocer cuál es la concepción del colectivo adolescente y si lo entienden como un aspecto a ser incluido en el currículo educativo. Por lo tanto, se han realizado preguntas de las cuales se derivan respuestas que confirman la inclusión de la temática en el contexto académico y la necesidad de que se forme, no solo al alumnado, sino también al profesorado. De tales afirmaciones se infiere la necesidad de modificar el currículo y la indispensable formación continua a la que se han referido, entre otros (Quendler y Lamb, 2016). Además, es necesario hacer hincapié en el alcance de la mirada crítica y el empoderamiento de los menores para que convivan de forma pacífica, respetuosa y responsable tanto en la presencialidad como en la virtualidad, una necesidad que se agrava en la etapa adolescente y se hace indispensable en un momento en el cual Internet, «facilita la tarea del acosador sobre el acosado» (Caldeiro-Pedreira, 2010).

En suma, se apela al diseño de metodologías y estrategias de intervención en situaciones de violencia (Cerezo, 2006), un objetivo que cumple el programa que se analiza y que se deriva de las afirmaciones de la muestra entrevistada. Además, se alza como una herramienta de prevención, por lo que su censura se convierte en un craso error ya que cumple una misión no solo informativa sino también formativa. Las emisiones de «Proyecto Bullying» han sido calificadas positivamente en la entrevista al considerar que la programación visibiliza una situación totalmente injusta e inexplicable y que les permite expresar a los participantes en el programa, como paso previo para afrontar y superar los hechos.

En definitiva, y ante la situación casi generalizada de acoso y después de analizar las respuestas de la muestra de la zona norte y sur de España, puede afirmarse que los chicos y chicas se sienten abrumados al constatar que los agresores actúan de forma gratuita y continuada sobre las víctimas llegando a señalar que los primeros «están enfermos»; igualmente reclaman soluciones ante tal lacra. Aquellos con edades más tempranas son los que presentan un alto grado de atenuación y vulnerabilidad frente al problema, definiéndose como débiles, buscando la ayuda de los mayores. Además, no siempre señalan que actúan en casos de «bullying», una afirmación debida, fundamentalmente, a que no han vivido de primera mano una situación concreta o porque no suelen mezclarse en situaciones problemáticas. En este sentido, la afirmación se opone a la idea de intervención (Garaigordobil, Martínez-Valderrey y Machimbarrena, 2017) pese a ello, según se deriva de las respuestas, los jóvenes que conocen casos de acoso señalan que es necesario intervenir para frenar tales injusticias. Una intervención que reclaman de forma marcada los entrevistados de la zona sur de España, mientras que en el norte subrayan su importancia y necesidad. Por tanto, la intervención se erige como una necesidad básica y, en el caso escolar el trabajo del docente y de la institución para contribuir a generar espacios en los cuales no solo se conciencie e informe sino que ha de formarse adecuadamente. Además, y según se aprecia en las respuesta 
de los y las entrevistadas, es clave el papel de programas como el que se analiza siempre y cuando surtan un efecto adecuado y si cumplen las funciones mencionadas. Una idea, esta última que refuerza el valor de los medios de comunicación y su doble función, informativa y formativa en la sociedad digital más reciente cuando de forma especial los móviles e Internet, «se han convertido en medios esenciales para la socialización de los adolescentes (Álvarez-García et al., 2015).

\section{Referencias bibliográficas}

Aguaded-Gómez, I., Caldeiro-Pedreira, M. Y.C., y RodríguezLópez, J. (2015). ¿Qué nos muestran las pantallas?: La mirada crítica adolescente en el marco de las industrias culturales y del pensamiento actual. Alteridad. Revista de Educación, 10, 8-20. DOI: 10.17163/alt.v10n1.2015.01

Álvarez-García, D., Barreiro-Collazo, A., y Núñez, J. (2017). Ciberagresión entre adolescentes: prevalencia y diferencias de género. Comunicar, 50, 89-97. DOI: https://doi.org/10.3916/ C50-2017-08

Álvarez-García, D; Dobarro, A. B., y Núñez, J. C, (2015). Validez y fiabilidad del Cuestionario de cibervictimización en estudiantes de Secundaria. Aula Abierta, 43, 32-38. DOI: http://dx. doi.org/10.1016/j.aula.2014.11.001

Avilés, J. M. (2009). Ciberbullying. Diferencias entre el alumnado de secundaria. Boletín de Psicología, 96, 79-96.

Baxter, L.A., y Babbie, E.R. (2003). The Basics of Communication Research. Belmont CA: Wadsworth Thomson Learning.

Caldeiro-Pedreira, M. C. (2010). Ciberbullying y medios audiovisuales. Responsabilidad ciudadana y formación. En J. Gazquez Pérez, J. y M.C. Pérez (2010): Actas del II Congreso Internacional de Convivencia Escolar (pp. 131-138). Granada: GEU.

Cerezo, F. (2006). Violencia y victimización entre escolares. El bullying: estrategias de identificación y elementos para la intervención a través del Test Bull-S. Revista electrónica de Investigación Psicoeducativa, 9, 4(2), 332-352.

Cerezo, F., Calvo, A., y Sánchez, C. (2011). El programa CIP para la intervención específica en bullying. Madrid: Pirámide.

Díaz-Aguado, M. J., y Falcón, L. (2006). Los medios de comunicación como herramienta para la prevención. El programa «prevenir en Madrid. Psicología, 12(2), 85.

Gámez-Guadix, M., Villa-George, F., y Calvete, E. (2014). Psychometric properties of the Cyberbullying Questionnaire (CBQ) among Mexican adolescents. In R. Maiuro (Eds.). Perspectives on Bullying: Research on Childhood, Workplace, and Cyberbullying (p.65-80). New York: Springir Publishing Company.

Garaigordobil, M., y Oñederra, J. A. (2010). Inteligencia emocional en las víctimas de acoso escolar y en los agresores. European Journal of Education and Psychology, 3(2), 243-256.

Garaigordobil, M. (2011). Prevalencia y consecuencias del cyberbullying: una revisión. International Journal of Psychology and Psychological Therapy, 11(2), 233-254.

Garaigordobil, M., Martínez-Valderrey, V., y Machimbarrena, J. M. (2017). Intervención en el bullying y cyberbullying: Evaluación del caso Martín. Revista de Psicología Clínica con Niños y Adolescentes, 4(1), 25-32.

García-Orza, J. (1995). Violencia interpersonal en la escuela. El fenómeno del matonismo. Boletín de Psicología, 49, 87-103.

Hernández-Granda, E. (2001). Agresividad y relación entre iguales en el contexto de la enseñanza primaria. Estudio piloto (Tesis Doctoral). Oviedo: Universidad de Oviedo.

Hinduja, S., y Patchin, J. W. (2007). Internet use control for parents and children/teenagers. Recuperado de https://goo.gl/al6q3i
Hernández-Serrano, M., Renés-Arellano, P., Graham, G., y Greenhill, A. (2017). Del prosumidor al prodiseñador: el consumo participativo de noticias [From Prosumer to Prodesigner: Participatory News Consumption]. Comunicar, 50, 77-88. DOI: https://doi.org/10.3916/C50-2017-07

Intef (2017). Marco común de competencia digital docente. Recuperado de https://goo.gl/GFOq3k

Lacasa, C. S., y Ramírez, F. C. (2017). Conceptualización del bullying y pautas de intervención en educación primaria. International Journal of Developmental and Educational Psychology. Revista INFAD de Psicología., 6(1), 443-452. DOI: http://dx.doi. org/10.17060/ijodaep.2014.n1.v6.764

Loredo-Abdalá, A., Perea-Martínez, A., y López-Navarrete, G. E. (2008). Bullying: acoso escolar. La violencia entre iguales. Problemática real en adolescentes. Acta Pediátrica de México, 29(4), 210-214.

Lumsdem, L. (2002). Preventing Bullying. Office of Educational Research and Improvement. Washington DC: LTC.

Nansel, T.; Overpeck, M.; Haynie, D.; Ruan, W., y Scheidt, P. (2003). Relationships between Bullying and Violence among US youth. Archives of Pediatrics y Adolescent Medicine, 157, 348353.

Olweus, D. (2013). School bullying: Development and some important challenges. Annual Review of Clinical Psychology, 9, 751-780.

Ortega-Barón, J. (2016). Influencia del clima escolar y familiar en adolescentes, víctimas de ciberacoso. Comunicar, 24(46), 5765. DOI: http://dx.doi.org/10.3916/C46-2016-06

Perry, D. G., Kusel, S. J., y Perry, L. C. (1988). Victims of peer aggression. Developmental Psychology, 245, 807-814.

Pepler, D. J., y Rubin, K. H. (1991). The development and treatment of childhood aggression. Hillsdale NJ: Lawrence Erlbaum.

Quendler, E., y Lamb, M. (2016). Learning as a lifelong process - meeting the challenges of the changing employability landscape: competences, skills and knowledge for sustainable development. International Journal of Continuing Engineering Education and Life Long Learning, 26(3), 273-293. DOI: http:// dx.doi.org/10.1504/IJCEELL.2016.078447.

Ramírez, S. (2006). El maltrato entre escolares y otras conductas problema para la convivencia: Un estudio desde el contexto del grupo-clase. Tesis doctoral inédita. Granada: Universidad de Granada.

Romera, E., Cano, J., García-Fernández, C., y Ortega-Ruiz, R. (2016). Cyberbullying: competencia social, motivación y relaciones entre iguales. Comunicar, 48, 71-79. DOI: https:// doi.org/10.3916/C48-2016-07

Sánchez-Carrero, J., y Contreras-Pulido, P. (2012). De cara al prosumidor. Producción y consumo empoderando a la ciudadanía 3.0. Icono 14, 10 (3).62-84. DOI: 10.7195/ri14.v10i3.210

Schultze-Krumbholz, A., Schultze, M., Zagorscak, P., Wölfer, R., y Scheithauer, H. (2016). Feeling cybervictims' pain. The effect of empathy training on cyberbullying. Aggressive Behavior, 42(2), 147-156. DOI: https://doi.org/10.1002/ab.21613

Smith, P. K., Mahdavi, J., Carvalho, M., Fisher, S., Russell, S., y Tippett, N. (2008). Cyberbullying: its nature and impact in secondary school pupils. Journal of Child Psychology and Psychiatry, 49, 376-385. DOI: http://dx.doi.org/10.1111/j.14697610.2007.01846.x

Torres-Toukoumidis, Á., Romero-Rodríguez, L. M., De-Casas-Moreno, P., y Aguaded, I. (2017). Construcción del discurso bélico desde las agencias internacionales de noticias: Estudio de Caso Atentados del 13 de noviembre de 2015. Revista Mediterránea de Comunicación, 8(1), 121-135. DOI: https:// doi.org/10.14198/MEDCOM2017.8.1.9 
Van Dijk, T. (1983). Discourse analysis: Its development and application to the structure of news. Journal of Communication, 33(2), 20-43. DOI: http://dx.doi.org/10.1111/j.1460-2466.1983. tb02386.x
Vivar, D. M. (2010). La escuela inclusiva ante el acoso escolar. Revista Educación Inclusiva, 3, 123-133. 